\title{
Heterochrony repolarized: a phylogenetic analysis of developmental timing in plethodontid salamanders
}

\author{
Ronald M Bonett*, Michael A Steffen and Grant A Robison
}

\begin{abstract}
Background: Disentangling evolutionary shifts in developmental timing (heterochony) is dependent upon accurate estimates of ancestral patterns. However, many classic assessments of heterochronic patterns predate robust phylogenetic hypotheses and methods for trait reconstruction, and therefore may have been polarized with untested 'primitive' conditions. Here we revisit the heterochronic modes of development that underlie the evolution of metamorphosis, maturation, and paedomorphosis in plethodontid salamanders. We focus on the tribe Spelerpini, which is a diverse clade that exhibits tremendous variation in timing of metamorphosis and maturation, as well as multiple independent instances of larval form paedomorphosis. Based on morphology and biogeography, early investigators concluded that the most recent common ancestors of plethodontids, and also spelerpines, were large salamanders, with very long larval periods and late maturation times. This prevailing assumption influenced subsequent heterochronic assessments, which concluded that most modern spelerpines (with shorter larval periods) were derived through multiple independent accelerations in larval development. It was also concluded that most occurrences of larval form paedomorphosis in this clade resulted from progenesis (acceleration of gonadal development relative to metamorphosis).
\end{abstract}

Results: By reconstructing the time to metamorphosis on a molecular-based phylogeny of plethodontids, we find that ancestral spelerpines likely had relatively shorter larval periods than previously proposed. Taken together with the credibility interval from our ancestral state estimation we show that very long larval periods are likely derived decelerations, only a few lineages have undergone appreciable accelerations in metamorphic timing, and the remaining taxa have lower probabilities of being different than the ancestral condition (possibly due to stasis). Reconstructing maturation age across nodes concomitant with the evolution of larval form paedomorphosis in one large radiation does not show clear evidence of progenesis, but more likely indicates a case of neoteny (delayed metamorphosis).

Conclusions: This study demonstrates cases in plethodontid salamanders where phylogenetic-based character reconstructions reject previously hypothesized ancestral life history conditions. As a result, several prior hypotheses of heterochronic evolution in this family are reversed.

Keywords: Ancestral state reconstruction, Caudata, Evolution, Life history, Neoteny, Paedomorphosis, Progenesis

\footnotetext{
* Correspondence: ron-bonett@utulsa.edu

Department of Biological Science, University of Tulsa, Tulsa, OK 74104, USA
} 


\section{Background}

Shifting the ontogenetic time of developmental events may be a primary mechanism for producing diversity of morphology and life history [1-6]. Disentangling specific heterochronic patterns can provide insight into the direction, developmental mechanisms, and ecological circumstances that promote evolutionary shifts [1-10]. However, conducting comparative analyses of heterochronic patterns can be challenging and easily confounded. This is because these analyses rely on knowledge of the timing of developmental events for multiple taxa, an understanding of their phylogenetic relationships, and methods to deduce ancestral patterns of development [11-19]. Evolutionary and developmental biologists have been interested in heterochronic patterns for several decades, with the greatest surge of papers over the last 35 years $[1,2,20]$. However, only recently have robust phylogenies of many clades come into focus, as well as adequate methods for reconstructing and testing ancestral states. This suggests that some non-phylogenetically (or non-paleontologically) polarized heterochronic assessments may need to be reconsidered in light of more refined phylogenetic hypotheses and/or ancestral state reconstruction methods.

The phenomenon of 'larval form paedomorphosis' in salamanders has been a hallmark example of heterochrony [1]. This developmental pattern occurs when adults retain suits of larval juvenile characteristics and aquatic ecology into adulthood, and has independently evolved multiple times in salamanders [21-25]. There are at least two general ways in which larval form paedomorphosis can occur: (1) neoteny, which is decelerating or delaying metamorphic changes (somatic morphogenesis) despite consistent timing 'of reproductive development compared to ancestors; and (2) progenesis, which is accelerating or advancing gonadal development relative to somatic development. In other words, maturation precedes metamorphosis, resulting in an adult with a larval morphology (Figure 1; see also Methods section for additional qualifications of terminology). Quantitative tests of whether neoteny or progenesis is responsible for paedomorphosis in salamanders have been restricted to a few intraspecific comparisons in facultatively paedomorphic species $[7,8]$. However, heterochronic analyses of metamorphosis and maturation have not been conducted in a phylogenetic context to test the developmental origins of larval form paedomorphosis for any salamander clades.

The Family Plethodontidae is the most species rich clade of salamanders [21] and exhibits a wide range of life history strategies [21-26,28]. These include aquatic larvae followed by metamorphosis (biphasic), larval form paedomorphosis, and the absence of free-living aquatic larval forms (direct development; Figure 1). The biphasic life history occurs in at least three lineages of plethodontids (desmognathines, spelerpines, and the monotypic genus Hemidactylium).
Given that most salamander families also have aquatic larvae, the first assessments of plethodontid evolution concluded that clades with biphasic taxa diverged early in the evolution of the family, and biphasic life history was the ancestral condition [29-31]. More specifically, based on morphology and biogeography, it was proposed that the ancestral 'hemidactyline' (spelerpines + Hemidactylium) was a large salamander, with a long larval period and late maturation time (similar to the genus Gyrinophilus [29-33]). Based on this hypothesis for the ancestral condition, subsequent estimates of heterochronic patterns in the 'hemidactylines' concluded that the shorter larval periods of most species groups evolved from multiple independent accelerations in larval development [26] (Figure 1).

This study [26] also concluded that most instances of larval form paedomorphosis in spelerpines (particularly the genus Eurycea) resulted from progenesis rather than neoteny (Figure 1). Since then, robust molecular phylogenies of plethodontids, have completely inverted our understanding of the directionality of life history evolution in this family with regard to direct development and the evolution of larval periods $[28,34,35]$. Furthermore, a recent phylogeny of spelerpines [25] has provided a framework for re-evaluating the modes of heterochrony in this clade, which includes all paedomorphic plethodontids. However, these studies analyzed the evolution of life history as a dichotomous trait (biphasic vs. direct development [28]; biphasic vs. paedomorphic [25]), and patterns of timing of metamorphosis and maturation have yet to be revisited.

Here we analyze patterns of heterochrony in plethodontids, by reconstructing ancestral larval periods and maturation times on a well-rooted phylogeny, and testing the fit of alternative ancestral states at key nodes. We use the credibility interval from continuous ancestral state estimation to further analyze patterns of acceleration and deceleration in a diverse clade (tribe Spelerpini). Furthermore, we re-evaluate the heterochronic patterns (neoteny vs. progenesis) that likely led to paedomorphosis in one large radiation of paedomorphic spelerpines from the Edwards Plateau of Central Texas. We show that, in light of the molecular phylogeny and ancestral state estimates, many previously hypothesized patterns of heterochrony in plethodontids are reversed. This study highlights the importance of ancestral state reconstruction and estimation limits for understanding patterns of heterochrony.

\section{Methods}

\section{Terminology}

We acknowledge that the history of heterochronic terminology has been tumultuous $[1,2,27]$. For comparative purposes, here we follow the terminology of Ryan and Bruce [26], which has been the only prior comprehensive treatment 


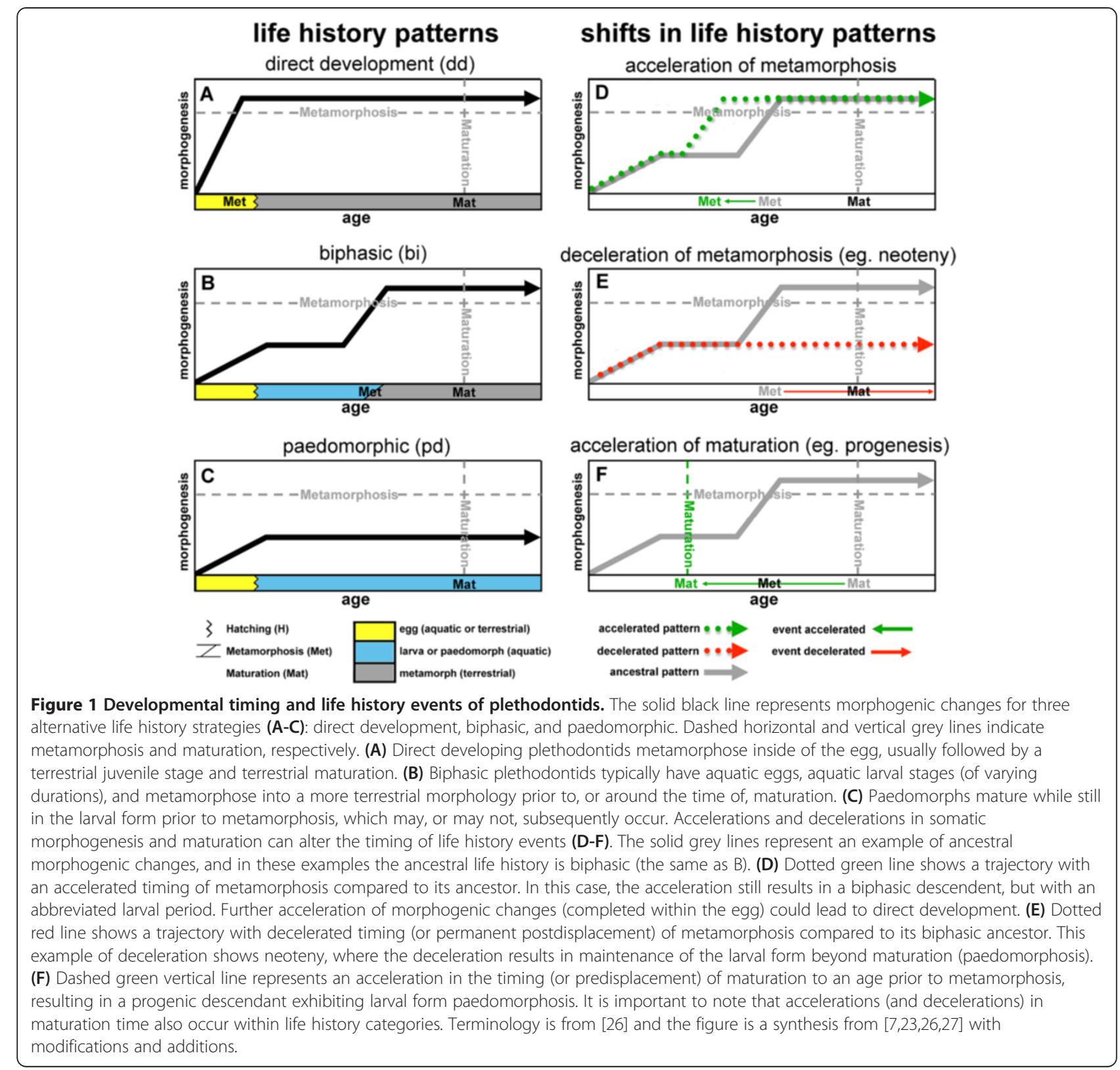

of heterochrony in spelerpine plethodontids. We use the terms acceleration and deceleration, respectively, to refer to the relative advancement and delay of the timing of developmental events compared to ancestors (Figure 1). These terms are applied to processes effecting somatic and reproductive tissues, which may, or may not, result in a shift between life history categories (direct development, biphasic, paedomorphic). For example, if metamorphosis of a biphasic species occurs significantly earlier than metamorphosis of its biphasic ancestor then this would be an acceleration in the age (timing) of metamorphosis.
Also following Ryan and Bruce [26] and other studies [8], we use the terms neoteny and progenesis primarily to refer to somatic deceleration and reproductive acceleration, respectively, which are processes that can result in larval form paedomorphosis. There are multiple ontogenetic trajectories that can lead to an advancement or delay of a developmental event. Since shifts in maturation and metamorphosis could be considered changes to the 'onset' or 'offset' of a developmental trajectory, then terms implying a 'rate' (neoteny, progenesis, acceleration, deceleration) may not apply. Instead, terms such as predisplacement and postdisplacement 
have been used to describe such shifts in the timing of reproduction and metamorphosis $[2,7]$.

\section{Data}

Data on timing of metamorphosis (age at metamorphosis) and maturation (age of gonadal maturation) in months for 63 plethodontids were primarily derived from the literature and some personal and unpublished observations by colleagues (Additional file 1). This sampling included representatives of most of the major lineages of plethodontids: 26 species of spelerpines from four of the five genera (21 Eurycea, 2 Gyrinophilus, 2 Pseudotriton, and 1 Stereochilus), 14 species of desmognathines (Desmognathus), and 23 other plethodontids (2 Aneides, 1 Batrachoseps, 2 Bolitoglossa, 1 Ensatina, 1 Hemidactylium, 1 Hydromantes, 14 Plethodon, and 1 Pseudoeurycea). Our analyses were based on minimum estimates of age at metamorphosis. Direct developing species metamorphose prior to hatching, so we considered their age at metamorphosis to be the time prior to hatching, which for most species was approximately 2 months [21]. Most paedomorphic plethodontids do not metamorphose (obligately paedomorphic), and this is an independently derived state in multiple lineages of spelerpines [25]. Since we were most interested in reconstructing the ancestral timing of metamorphosis, we coded paedomorphic taxa as missing metamorphic data in our analyses of metamorphic timing. We dealt with the evolution of larval form paedomorphosis (compared to direct development or biphasic) in a separate analysis (described below).

We also used minimum age estimates for maturation for all 63 species, and we analyzed male and female maturation times separately. We used minimum age (as opposed to average or maximum age) because it is the most consistent and obtainable metric across species. Most referenced studies are based on evaluating gonadal development across age/size classes. Therefore, we used minimum age at gonadal maturation (which is observed morphologically), as opposed to age at first reproduction (oviposition or spermatophore drop), which are less commonly documented. For example, the minimum age of reproductive maturation for both male and female Desmognathus ocoee has been documented at 3 years $[36,37]$. Even though most female $D$. ocoee may not oviposit until year 4, we used 3 years to be consistent with other studies that are only based on gonadal maturation.

There have been phylogenetic based reconstructions of plethodontid life history: biphasic vs. direct development [28] and biphasic (metamorphic) vs. paedomophic [25]. However, these three states have not been reconstructed in the same analysis. Therefore, we also reconstructed ancestral life history (direct development, biphasic, paedomorphic) for 100 plethodontids, including all North
American and Eurasian genera, as well as a newly described paedomorphic species (E. subfluvicola [38]). Life history information for these species is well established and was taken from the literature (Additional file 1). We only included three representative genera from the tropical radiation (bolitoglossines), due to the limited number of lengthy Rag1 sequences available for this group (see below), but it is clear that this radiation is monophyletic, and all species are thought to be direct developers. In other words, including additional bolitoglossines to our analyses would not significantly change the results presented here. The purpose of this analysis was primarily to reconstruct the origins of paedomorphosis within spelerpines, which was necessary for subsequent tests of progenesis $v s$. neoteny (see below). However, we also performed additional life history reconstructions and included the outgroup families Amphiumidae and Rhyacotritonidae to further test the ancestral life history mode of plethodontids (see Results).

\section{Phylogeny}

We reconstructed two chronograms of plethodontids which included representatives of: (1) all 100 taxa for ancestral life history analysis; and (2) the 63 taxa for which we have data on timing of metamorphosis and maturation. The chronograms were each based on complete datasets of 1,033 bp of the recombination activating gene 1 (Rag1; Additional file 1). Rag1 was chosen because it is a conserved nuclear locus that was already available for most taxa included in this study, and provides a close approximation to the topologies and branch lengths of previously reconstructed salamander phylogenies [25,28,34,39-43]. The sequences were primarily derived from previous phylogenetic datasets of plethodontids [28], spelerpines [25], Plethodon [44], and additional sequences for nine species from the genus Desmognathus that we collected for this study (Additional file 2).

Sequences were aligned using Sequencher v. 4.8 (Gene Codes, Ann Arbor, MI, USA), and the alignment was unambiguous with no missing data. MrModeltest v. 2.2 [45] was used to determine the most appropriate model of nucleotide substitution for each codon position (Additional file 3). The chronograms were estimated using BEAST v. 1.6 [46]. We applied the best-fitting models determined above, and the analysis was based on an uncorrelated lognormal molecular clock and Yule speciation prior across the tree. The fossil record of plethodontids is very limited $[47,48]$, so we used the base of the crown group of extant plethodontids as a calibration point. The estimates of the deepest divergence for this clade are in the range of 41 Mya to 99 Mya, with average estimates at approximately 73 Mya [40,41,49-51]. We applied a normally distributed calibration prior for the crown group of plethodontids, with a mean of 73 Mya and standard deviation of 6 Mya. 
This combination of parameters yielded a $95 \%$ prior distribution between 85 Mya and 65 Mya, representing a reasonable range of potential dates for this clade based on previous studies. Analyses are based on relative branch lengths of the chronograms, and would be the same regardless of the overall time scale. Both analyses were run twice independently for 20 million generations with trees saved every 1,000 generations (total 40,000 trees). Likelihood values across generations were evaluated in Tracer v. 1.5 [52] and the first $25 \%$ of generations from both runs (10,000 trees) were conservatively discarded as burnin, which was well beyond stationarity. Both chronograms (100 taxa and 63 taxa) were similar in branch lengths and topology. We used the 30,000 post-burnin trees, from the phylogenetic analysis of each dataset, for their respective reconstructions (see below).

\section{Ancestral state reconstruction}

Ancestral life histories, ages of minimum metamorphosis, and ages of minimum maturation (males and females) of plethodontid salamanders were reconstructed using Bayesian methods. Categorical and continuous ancestral reconstructions were performed in BayesTraits v. 2.0 [53] using 'BayesMulitState' [54] and a Markov Chain Monte Carlo (MCMC) model. Reconstructions were based on all 30,000 post-burnin Bayesian chronograms from the phylogenetic analysis in BEAST. Uniform priors from 0 to 100 were applied for each analysis, and acceptance rates were between $20 \%$ and $40 \%$. Each analysis was run for 5 million generations with samples taken every 1,000 generations, with the first 1 million generations of each run discarded as burnin (that is, ancestral state results were based on 4 million post-burnin generations $=4,000$ samples).

Life history was reconstructed as an ordered, categorical trait with three states (direct development, biphasic, paedomorphic). Age-based traits (metamorphosis and maturation) were analyzed using both a 'continuous' (number of months) and categorical coding (number of years). The categorical analyses allowed for testing among alternative states for some key ancestral nodes (for example, the age of metamorphosis and maturation for the clade Spelerpini; described below). We divided continuous ages into four metamorphic age categories: $1=11$ months or less; $2=12$ to 23 months; $3=24$ to 35 months; $4=36$ months or more. A similar strategy was applied for both minimum male and female maturation, but included two additional age categories: $1=11$ months or less; $2=12$ to 23 months; $3=24$ to 35 months; $4=36$ to 47 months; $5=48$ to 59 months; $6=60$ months or more.

For life history reconstructions, transitions were only allowed between biphasic and direct development or biphasic and paedomorphic, but not between direct development and paedomorphic (transitions set to zero probability). Likewise, transitions between categorical age states were also ordered numerically by setting nonnumerically adjacent categories to zero probability. For example, for the four metamorphic age categories, transitions were allowed in both directions between categories 1 and 2, 2 and 3, and 3 and 4, but not between 1 and 3,1 and 4, or 2 and 4. The same strategy was applied to the six maturation age categories for males and females. Implementing ordered categories enforces ancestors to sequentially evolve through age categories (without skipping), and it also reduces the number of possible transitions for our reconstructions. Our analyses with ordered age categories were always a better fit than analyses with unrestricted transitions between categories.

All ordered transitions between states (within traits) were set to equal rates (that is, one-rate models). For each trait we compared the fit of a one-rate model to a model where transition rates (for ordered states) were allowed to vary (multi-rate models). The lowest AIC score indicates the best fitting model. $\triangle \mathrm{AIC}$ values $<3$ were considered to be negligible differences between models, values $\geq 3$ were considered moderately strong, and values $\geq 10$ were considered very strong support for rejecting the alternative model with the higher AIC score [55]. For each trait, a one-rate model was a substantially better fit than the multi-rate model (Life history $\triangle \mathrm{AIC}=21.62$; Metamorphic Age $\triangle \mathrm{AIC}=8.59$; Male Maturation Age $\triangle \mathrm{AIC}=9.12$; Female Maturation Age $\triangle \mathrm{AIC}=27.08)$.

We used BayesTraits to test for differences among ancestral conditions for key nodes in the phylogeny of spelerpines. These analyses were performed by fixing ('fossilizing') nodes to alternative states and comparing harmonic means (hm) for each run by calculating differences in Log Bayes Factors (LBf). The lowest LBf indicates the best fitting model $[53,54]$. LBf values $<3$ were considered negligible differences between models, values $\geq 3$ show were considered moderately strong, and values $\geq 10$ were considered very strong support for rejecting the alternative model with the higher LBf.

Continuous trait analyses of the timing of metamorphosis, and male and female maturation were performed in BayesTraits [53,54] using MCMC under a Brownian Motion ('Continuous Random Walk') model. Reconstructions were based on all 30,000 post-burnin Bayesian chronograms from the phylogenetic analysis in BEAST. Run generation parameters were the same as described above for multistate analyses, and results were based on 4,000 post-burnin samples.

We used the Bayesian 95\% Highest Prior Density (HPD) credibility interval of the ancestral state of spelerpines to determine which taxa have metamorphic ages that arose from acceleration (less than the 95\% HPD interval), deceleration (greater than the 95\% HPD 


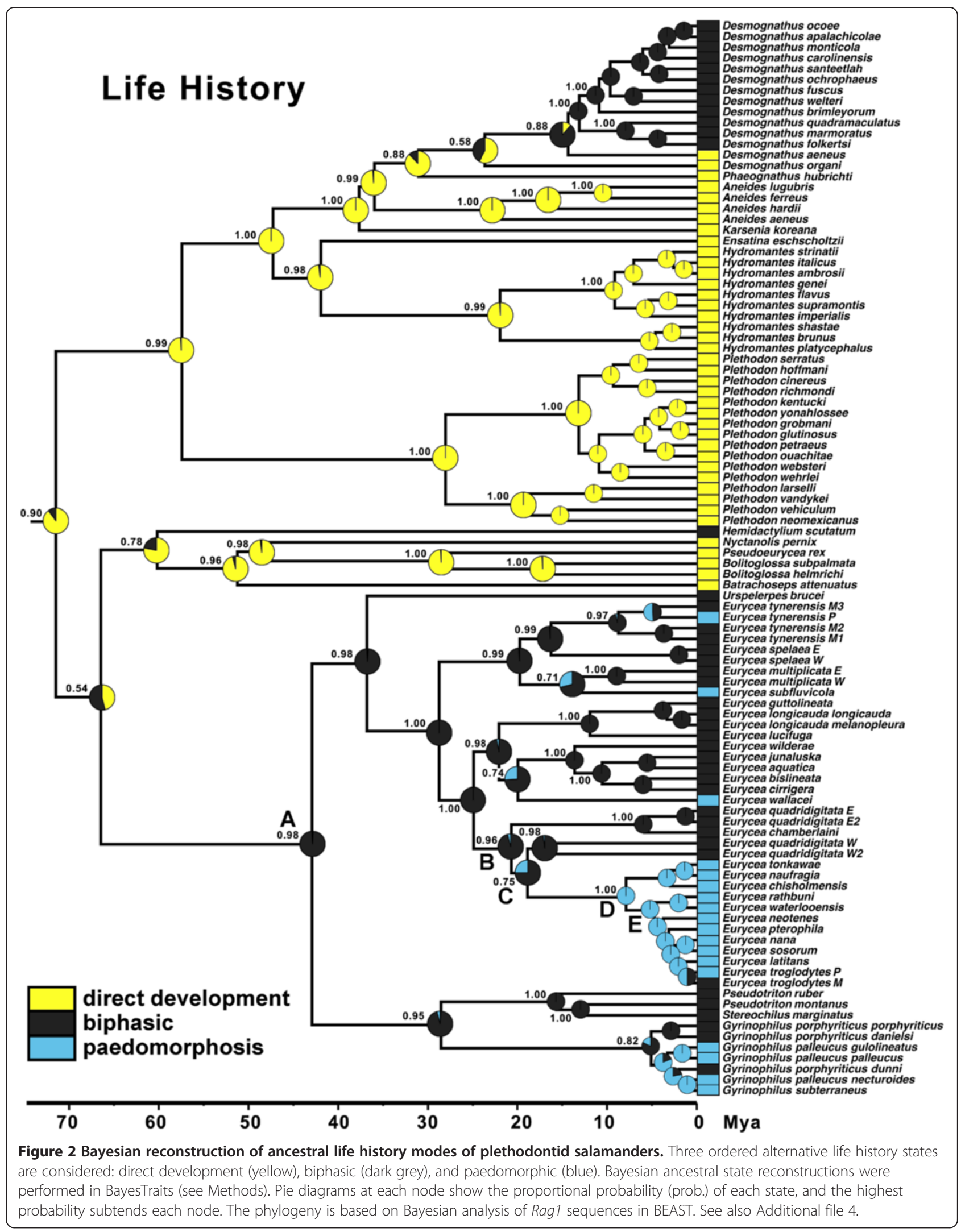


interval), or stasis (within the 95\% HPD interval; in other words, showing a lower probability of being different than our ancestral state estimate). Additionally, to test if larval form paedomorphosis arose from neoteny or progenesis, we examined timing of maturation across the evolutionary shift from metamorphosis to paedomorphosis in a large clade of paedomorphic Eurycea from the Edwards Plateau of Central Texas (Figure 2; node D). If paedomorphosis arose via progenesis (early maturation $[1,26])$, we would expect a significant reduction in maturation time concomitant with the evolution of paedomorphosis. In contrast, if paedomorphosis arose from neoteny (delayed somatic development $[1,26]$ ) then we would not expect significant differences in ancestral maturation patterns during the transition from metamorphosis to paedomorphosis. We quantified significant changes in ancestral maturation (for males and females separately using both categorical and continuous analyses). For the categorical analyses we fixed the ancestral states at four nodes spanning the evolution of paedomorphosis in Edwards Plateau Eurycea (Figures 2; nodes $B$ to $E)$ to the six alternative maturation categories (years). For a given node we used Log Bayes factors to compare which of the maturation category is the best fit, and which categories were significantly worse (methods described above). Again, for progenesis we would expect that the best fitting maturation ages would shift to younger age categories across these nodes, whereas neoteny should show no change (or an increase) in maturation age categories. We further compared the 95\% HPD interval of continuous maturation age reconstructions from BayesTraits (above) for these nodes (B to E) to evaluate potential reductions in maturation time (progenesis). Male and female maturation was analyzed separately for both categorical and continuous methods.

\section{Results}

\section{Reconstructions of life history mode}

Reconstruction of the ancestral life history mode of plethodontids shows strong support for a direct developing ancestor for the family (prob. $=0.90$; Figure 2). Fixing the common ancestor of plethodontids to direct development is a substantially better fit than biphasic $(\mathrm{LBf}=5.05)$ or paedomorphic $(\mathrm{LBf}=16.15)$. Inclusion of outgroup families (Amphiumidae and Rhyacotritonidae) also strongly supports a direct developing ancestor for plethodontids (prob. $=0.89$; Additional file 4). Among salamanders, direct development is unique to plethodontids, and biphasic is likely ancestral for salamanders $[22,23]$. Previous analyses have shown that Desmognathus with biphasic life histories are phylogenetically nested among direct developing taxa, so free living larval stages of this clade likely result from a reversal in developmental timing [28]. Our reconstructions show that biphasic life histories in spelerpines and Hemidactylium are also reversals to a free-living larval stage (Figure 2). Therefore, our reconstruction supports at least three independent reversals from direct development to a biphasic life history. It should be noted that some direct developing species retain ancestral larval anatomy during development (such as larval epibranchial cartilages) [34], so we can only consider these reversals in developmental timing, or reversals to a free living larval stage, but not necessarily reversals in larval anatomy.

Similar to recent analyses [25], biphasic (metamorphic) is the ancestral state for spelerpines (prob. $=0.98$; Figure 2; Table 1; Additional file 4), and there have been multiple independent instances of paedomorphosis in this clade. One important transition for our analyses of heterochronic patterns is the transition from metamorphosis to paedomorphosis in Eurycea from the Edwards Plateau of central Texas (taxa descending from node D; Figure 2).

Table 1 Tests of alternative ancestral life history modes for select plethodontid nodes

\begin{tabular}{llll}
\hline Node / Life history & Prob. & hm & LBf \\
\hline Root: Plethodontidae & & & \\
Direct development & 0.90 & -44.29 & 0.00 \\
Biphasic & 0.10 & -46.82 & 5.05 \\
Paedomorphic & 0.00 & -52.36 & 16.15 \\
Node A: Spelerpini & & & \\
Direct development & 0.01 & -51.65 & 15.35 \\
Biphasic & 0.98 & -43.98 & 0.00 \\
Paedomorphic & 0.01 & -51.52 & 15.09
\end{tabular}

Node B: Eurycea quadridigitata + Edwards Plateau Eurycea

$\begin{array}{llll}\text { Direct development } & 0.00 & -55.13 & 21.74 \\ \text { Biphasic } & 0.96 & -44.26 & 0.00 \\ \text { Paedomorphic } & 0.04 & -51.07 & 13.65\end{array}$

Node C: western Eurycea quadridigitata + Edwards Plateau Eurycea

\begin{tabular}{lccl} 
Direct development & 0.00 & -55.49 & 22.55 \\
Biphasic & 0.75 & -44.22 & 0.00 \\
Paedomorphic & 0.25 & -49.65 & 10.86 \\
\multicolumn{2}{l}{ Node D: Edwards Plateau Eurycea } & & \\
Direct development & 0.00 & -59.97 & 31.26 \\
Biphasic & 0.00 & -48.95 & 9.23 \\
Paedomorphic & 1.00 & -44.33 & 0.00
\end{tabular}

Node E: southern Edwards Plateau Eurycea

\begin{tabular}{llll} 
Direct development & 0.00 & -65.99 & 43.89 \\
Biphasic & 0.00 & -53.00 & 17.92 \\
Paedomorphic & 1.00 & -44.04 & 0.00 \\
\hline
\end{tabular}

Proportional probabilities are from Bayesian reconstructions of three ordered life history states (direct development, biphasic, and paedomorphic; Figure 2). Model fitting comparisons were performed by fixing ('fossilizing') select nodes to the three alternative life history states and comparing Log Bayes Factors (LBf) to the lowest (best fitting) life history for the node. 
We find that this transition most likely occurred between node C (biphasic prob. 0.75) and node D (paedomorphic prob. $=1.00)$. The biphasic to paedomorphic transition along this branch (between nodes $C$ and $D$ ) is further supported by fixing these nodes to each of the alternative life history states (Table 1). For node $\mathrm{C}$, biphasic is a substantially better fit than paedomorphic $(\mathrm{LBf}=10.88)$ and direct development $(\mathrm{LBf}=22.55)$. In contrast, paedomorphic is a substantially better fit for the life history of node D, compared to biphasic $(\mathrm{LBf}=9.23)$ or direct development $(\mathrm{LBf}=31.26)$.

\section{Reconstructions of metamorphic age}

Metamorphic age is highly variable across plethodontids (Figure 3). Consistent with life history mode reconstructions (Figure 2), ancestral plethodontids also show early metamorphic age: 0 to 11 months (categorical prob. = 0.81 ) or 7.6 months (continuous). The 0 to 11 month age category for ancestral plethodontids (root node) is a better fit than all other age categories (Additional file 5). This estimated age of metamorphosis is within the range of hatching time for direct developers. There were two major shifts to longer metamorphic ages (Figure 3). One shift is within the genus Desmognathus (quadramaculatus species group) and the other is in the lineage leading to spelerpines. The shift in metamorphic age of spelerpines is consistent with shifts from direct development to biphasic life histories (Figure 2).

We estimate that ancestral spelerpines likely had a larval period of 12 to 23 months (categorical prob. 0.50) or 16.0 months (continuous). This is a better fit than older, but not younger, age categories (Additional file 5). The 95\% HPD interval for our continuous metamorphic age estimate is 7.1 to 25.1 months (Figure 4; Additional file 6). Metamorphic age is highly variable among biphasic spelerpines. If we consider the 95\% HPD interval for ancestral metamorphic age, then the long larval periods of Gyrinopilus porphyriticus ( $\geq 36$ months) and Pseudotriton ruber (27 months; Figure 4), have a high probability of being decelerations in metamorphic timing. In comparison, E. quadridigitata, E. longicauda, and E. guttolineata, which minimally metamorphose in 5 months or less, have a high probability of being accelerations. All other spelerpine taxa fall within the 95\% HPD interval of our ancestral state estimate, and have lower probabilities of being different from the common ancestor of spelerpines (clade A). This may reflect either undetectable shifts (using this method and criterion) or stasis in metamorphic age.

\section{Reconstructions of maturation age}

Minimum age at maturation also varies extensively across plethodontids (Figures 5 and 6). We estimate that ancestral male spelerpines matured at 24 to 35 months (categorical prob. 0.39) or 30.9 months (continuous). Female spelerpines also likely matured at 24 to 35 months (categorical prob. 0.27) or 34.6 months (continuous). Later maturation ages are derived in Gyrinophilus, Pseudotrition, and Stereochilus, whereas maturation ages are reduced in some clades of Eurycea.

The earliest maturation times among plethodontids are seen in Edwards Plateau Eurycea, paraphyletic $E$. quadridigitata, and the common ancestors of these lineages (nodes B, C, and D). Categorical analyses support maturation ages between 0 to 11 months for males $(\mathrm{B}$ prob. $=0.77$; $\mathrm{C}$ prob. $=0.75 ; \mathrm{D}$ prob. $=0.64)$ and females $(\mathrm{B}$ prob. $=0.67 ; \mathrm{C}$ prob. $=0.68 ; \mathrm{D}$ prob. $=0.61)$. When we fixed these nodes ( $B$ to $D$ ) to alternative age categories, we find that for females the 0 to 11 age category is at least a moderately better fit than older maturation age categories (Table 2). Categorical estimates do not support a significant decrease in maturation age during the life history transition from biphasic to paedomorphic (between nodes C and D; Figure 7). Continuous estimates of maturation age for these nodes are also young, but consistently older than categorical estimates for both males (node $B=19.9$; node $C=15.8$; node $\mathrm{D}=14.8$; Figure 5 ) and females (node $\mathrm{B}=19.2$; node $C=16.2$; node $D=15.6$; Figure 6 ), with a high degree of overlap in these intervals. The estimated average maturation ages during the life history transition from biphasic to paedomorphic (between nodes $\mathrm{C}$ and D; Figure 7) also change very little ( 1 month decrease for males and 2.6 month decrease for females). Given that E. quadridigitata metamorphose in less than 6 months, and the estimated metamorphic ages for the common ancestors of this clade are 1 year or less (Figure 3; Additional file 6), then maturation would had to have advanced to a very early age in order to precede metamorphosis and achieve paedomorphosis via progenesis.

\section{Discussion}

\section{Repolarizing heterochronic shifts in plethodontids}

Previous investigations of heterochronic patterns of metamorphosis and maturation for plethodontid salamanders predate robust molecular phylogenetic hypotheses [26]. Ancestral plethodontids and spelerpines were posited to be large salamanders with long larval periods and late maturation times. This led subsequent investigators to conclude that shorter larval periods of most extant metamorphic taxa were derived through multiple independent accelerations of larval development. Here we show that by reconstructing the minimum age of metamorphosis on the phylogeny of plethodontids, that ancestral spelerpines likely had relatively short, as opposed to very long, larval periods (Figure 3). When we consider the credibility interval of our estimate of ancestral metamorphic age, and the metamorphic ages of descending lineages, then it is clear 


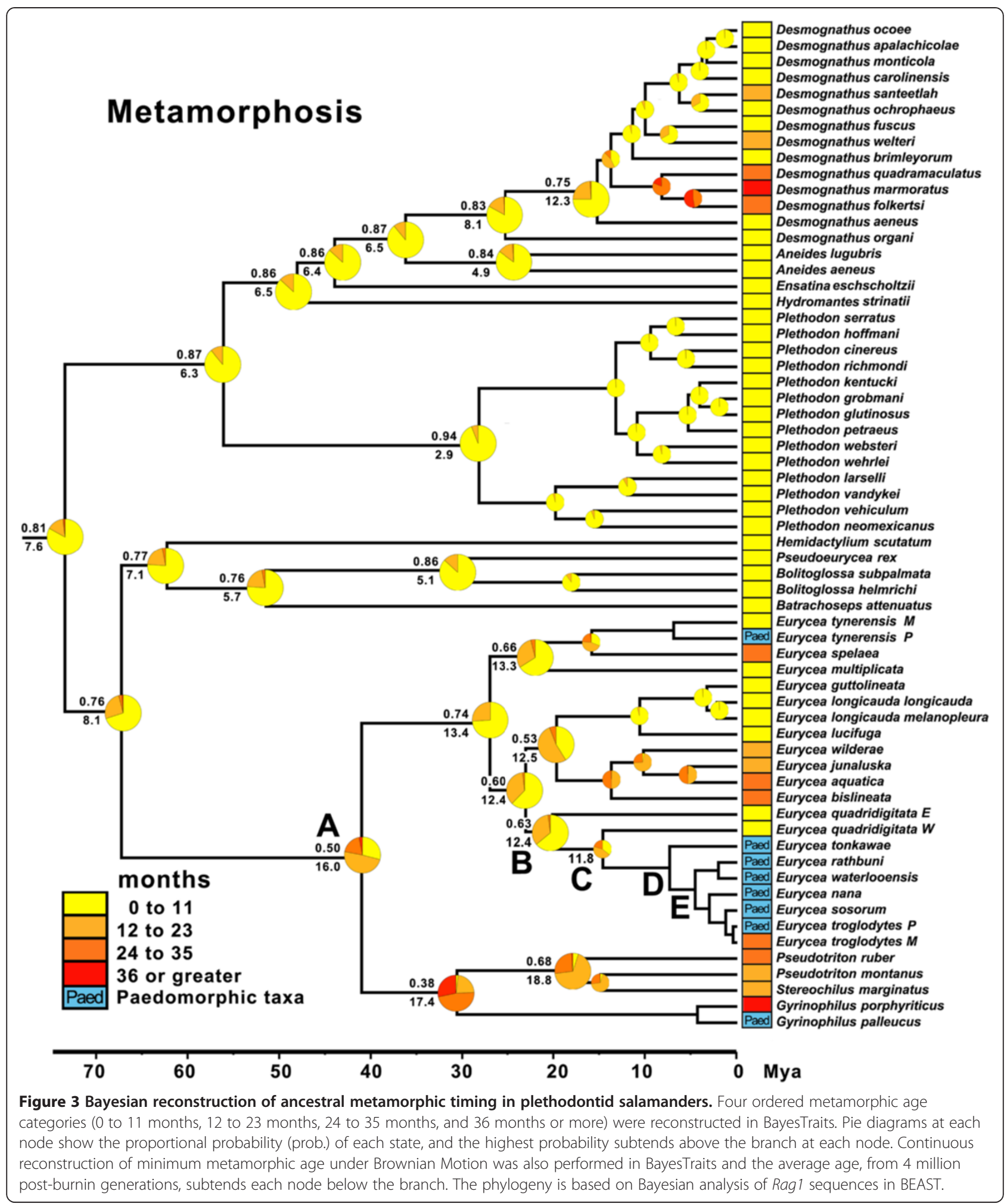

that very long larval periods (for example, G. porphyriticus and $P$. ruber) are derived decelerations. Only a few taxa show evidence of accelerations in metamorphic timing compared to ancestral spelerpines (E. quadridigitata, E. longicauda, and E. guttolineata), and the rest of the taxa are either relatively unchanged since the ancestral state (stasis) or have not changed enough to detect a herterochronic shift using these methods (Figure 4). 


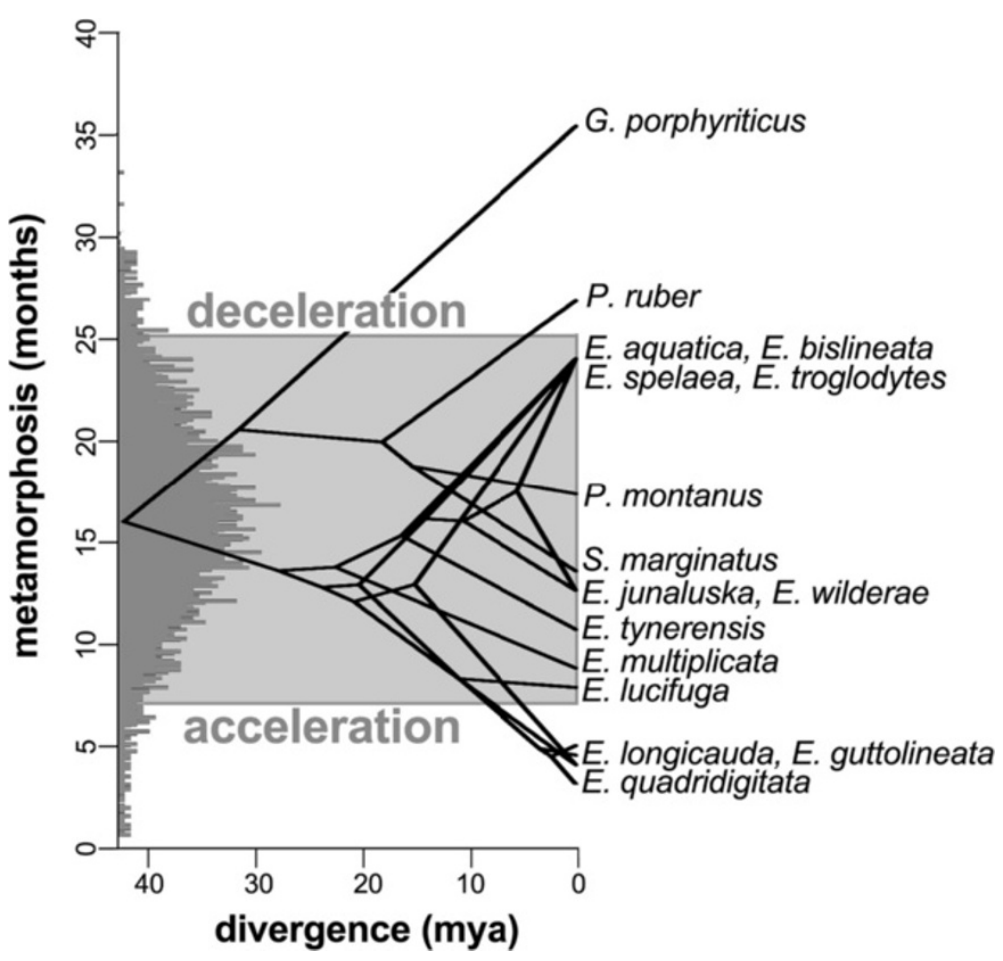

Figure 4 Phenogram of metamorphic timing of spelerpine plethodontids. The phenogram was reconstructed using phytools [56] under Brownian Motion (ancestral states are similar to those reconstructed using a Brownian Motion model in BayesTraits). The histogram is the Bayesian posterior samples from the ancestral metamorphic age estimate for spelerpines (average 16.0 months). The grey box indicates the $95 \%$ HPD interval of this estimate. Taxa above the box are considered decelerations in larval period compared to ancestral spelerpines. Taxa below the grey box are considered accelerations in larval period compared to ancestral spelerpines. Taxa within the grey box are within the range of our estimated credibility interval for the most recent common ancestor of spelerpines, and potentially represent stasis in metamorphic timing.

There are multiple pathways that can lead to the evolution of larval form adult descendants (paedomorphs) from metamorphic ancestors (Figure 1). Two general mechanisms are progenesis, which acts through the acceleration (or predisplacement) of reproductive development relative to somatic development, and neoteny, which is the delay (or postdisplacement) of somatic development relative to reproductive development ([1,2,26,57]; Figure 1). Most assessments and tests of progenesis $v s$. neoteny have been performed by observing or manipulating maturation and metamorphosis within facultatively paedomorphic species $[7,8,26]$. However, these traits have not previously been reconstructed in a phylogenetic context to test hypotheses about origins of paedomorphosis for major lineages. Larval form paedomorphosis has evolved independently, multiple times within spelerpine plethodontids (Figure 2). Previous heterochronic interpretations, performed prior to molecular phylogenetic estimates of spelerpines, concluded that paedomorphosis evolved via delayed metamorphosis (neoteny) in Gyrinophilus [58], and accelerated reproduction (progenesis) in Eurycea $[26,59,60]$. A progeneic mechanism for paedomorphic evolution in Eurycea from the Edwards Plateau of Central Texas (our clade D) was based on the notion that this clade was most closely related to two-lined salamanders (Eurycea bislineata group) from the southern Appalachian Mountains, which have older maturation ages (approximately 3 years) and relatively long larval periods for a Eurycea (approximately 2 years). This older maturation age was assumed to have been ancestral, and therefore the younger maturation ages of Edwards Plateau Eurycea (for example, E. neotenes) were considered an acceleration of maturation relative to metamorphosis (progenesis). However, recent phylogenetic analyses show that Edwards Plateau Eurycea are phylogenetically nested among dwarf salamanders (Eurycea quadridigitata) $[25,61]$, which have very short larval periods and maturation times more similar to Edwards Plateau Eurycea. Interestingly, the Eurycea quadridigitata group (clade B), which includes Edwards Plateau Eurycea, shows early maturation (progenesis) compared to other Eurycea and other plethodontids (Figures 5 and 6). Nevertheless, our reconstructions do not show evidence for major advancements in maturation age during the somatic shift from biphasic (metamorphic) to larval form paedomorphic development in Edwards Plateau Eurycea (ancestral nodes $\mathrm{C}$ to D; Figure 7). Therefore, even though the Eurycea quadridigitata group (clade B) may be generally progenic (compared to other plethodontids), we conclude 


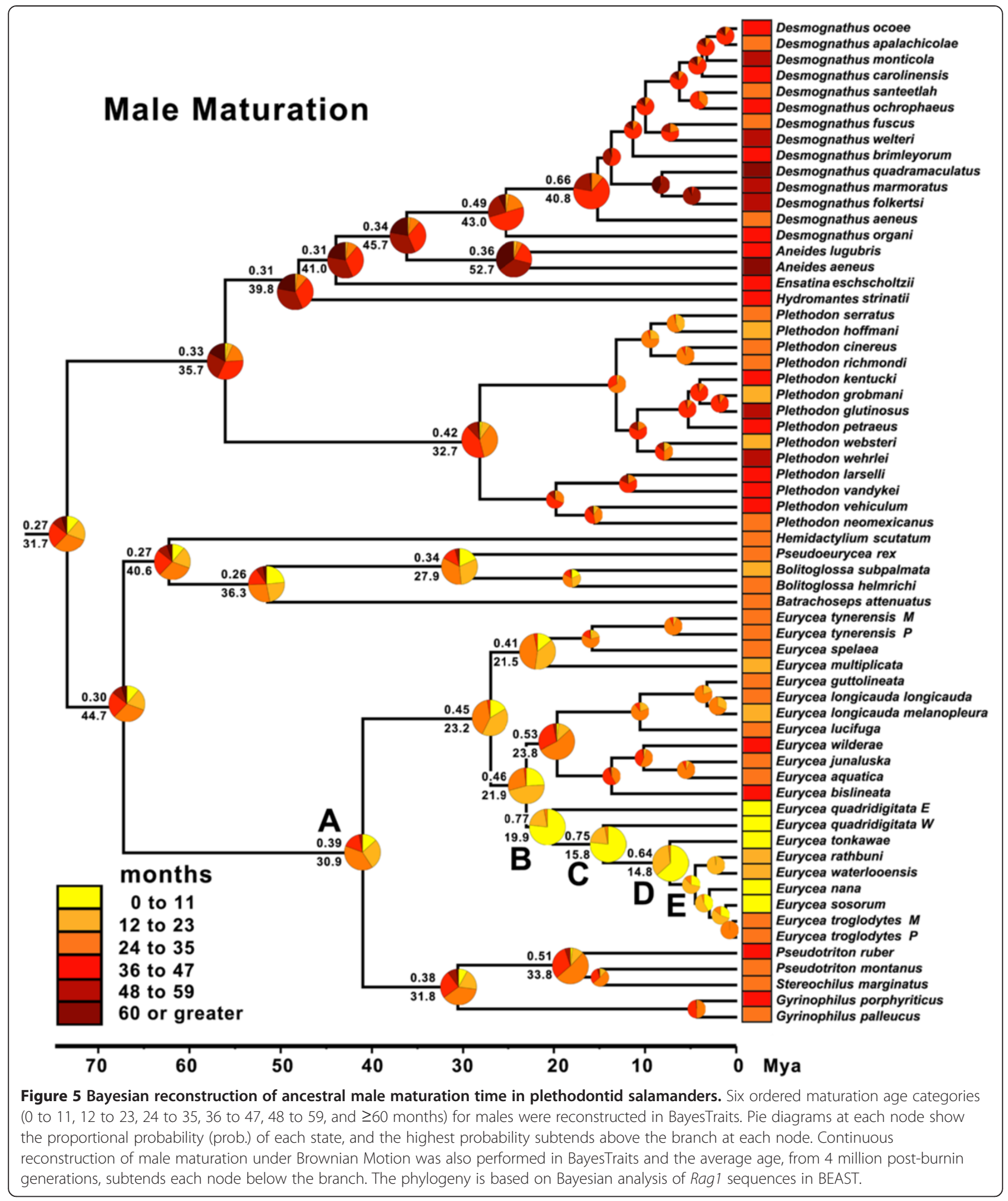

that the evolution of paedomorphosis in Edwards Plateau Eurycea (clade D) is due to neoteny (a somatic delay of metamorphosis without a shift in maturation age). It is important to note that some studies of facultatively paedomorphic salamanders show that predisplacement (progenesis) of maturation by approximately one to two months can lead to larval form paedomorphosis [7]. The macroevolutionary methods we employed here may not 


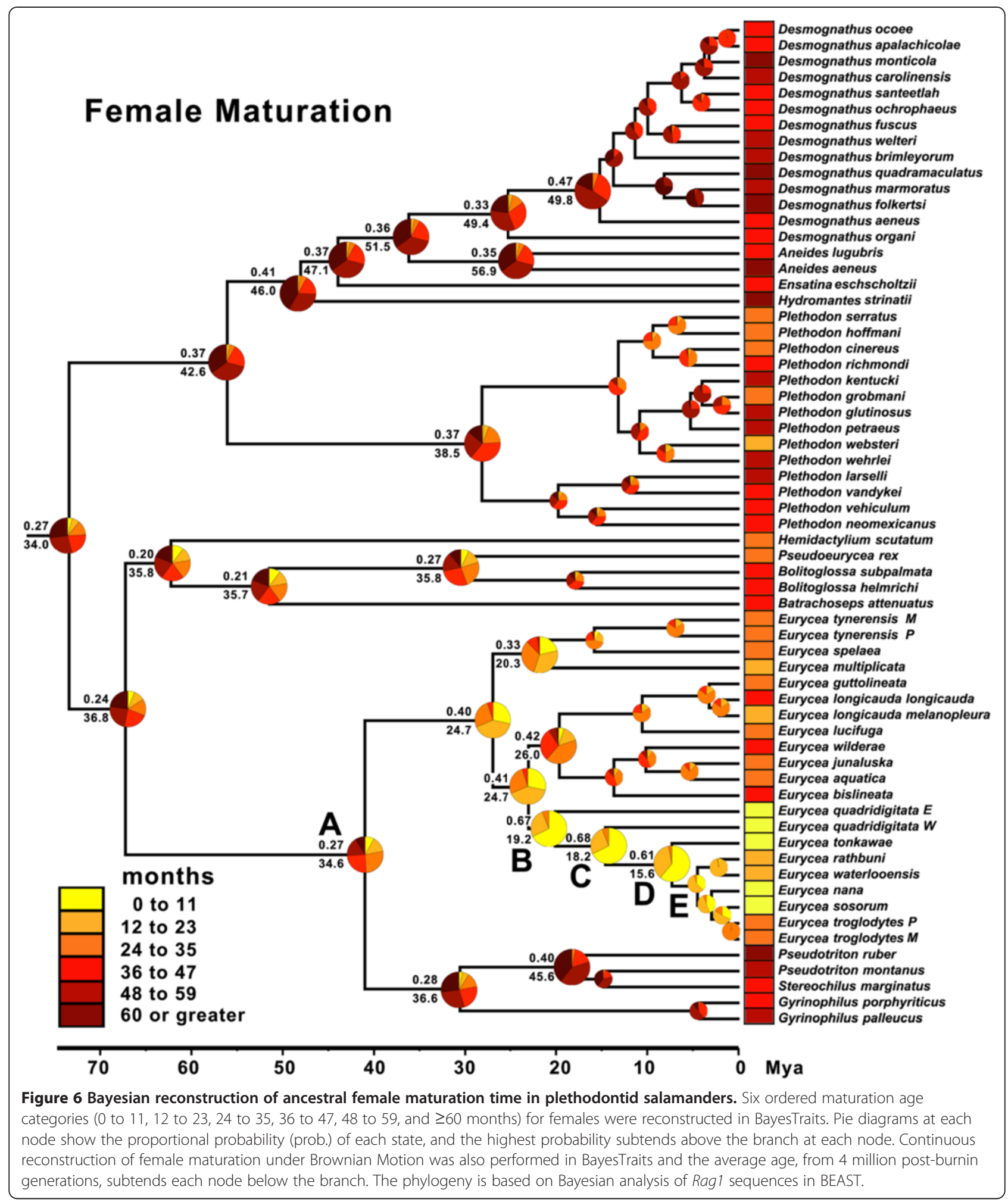

be sensitive enough to detect such a subtle shift in maturation age. However, metamorphosis of E. quadridigitata and the common ancestors of this clade (nodes B and $\mathrm{C}$ ) are fairly accelerated (Figures 3 and 4; Additional file 6), so maturation would have had to be considerably advanced to precede metamorphosis in this clade. More data on maturation age are needed for additional lineages of Eurycea, to further test if neoteny is a generalizable 
Table 2 Tests of timing of maturation for select plethodontid nodes (months)

\begin{tabular}{|c|c|c|c|c|c|c|}
\hline Node/Mat Age & M prob & M hm & M LBf & F prob & $\mathrm{F} \mathrm{hm}$ & F LBf \\
\hline \multicolumn{7}{|c|}{ Node A: Spelerpini } \\
\hline 0 to 11 & 0.14 & -106.89 & 10.63 & 0.08 & -105.06 & 9.80 \\
\hline 12 to 23 & 0.28 & -104.19 & 5.21 & 0.14 & -102.58 & 4.84 \\
\hline 24 to 35 & 0.39 & -101.58 & 0.00 & 0.27 & -102.08 & 0.00 \\
\hline 36 to 47 & 0.16 & -104.97 & 6.78 & 0.25 & -100.15 & 3.85 \\
\hline 48 to 59 & 0.03 & -105.72 & 8.27 & 0.17 & -102.60 & 4.88 \\
\hline$\geq 60$ & 0.00 & -107.79 & 12.43 & 0.09 & -102.72 & 5.13 \\
\hline
\end{tabular}

Node B: Eurycea quadridigitata + Edwards Plateau Eurycea

$\begin{array}{lllllll}0 \text { to } 11 & 0.77 & -100.86 & 0.00 & 0.67 & -101.08 & 0.00 \\ 12 \text { to } 23 & 0.20 & -101.57 & 1.40 & 0.27 & -103.66 & 5.15 \\ 24 \text { to } 35 & 0.03 & -102.59 & 3.45 & 0.06 & -104.03 & 5.90 \\ 36 \text { to } 47 & 0.00 & -109.53 & 17.33 & 0.00 & -105.84 & 9.52 \\ 48 \text { to } 59 & 0.00 & -112.52 & 23.31 & 0.00 & -110.84 & 19.52 \\ \geq 60 & 0.00 & -112.69 & 23.65 & 0.00 & -112.07 & 21.98\end{array}$

Node C: western Eurycea quadridigitata + Edwards Plateau Eurycea

$\begin{array}{lllllll}0 \text { to } 11 & 0.75 & -100.58 & 0.00 & 0.68 & -99.76 & 0.00 \\ 12 \text { to } 23 & 0.22 & -101.17 & 1.18 & 0.27 & -101.37 & 3.21 \\ 24 \text { to } 35 & 0.03 & -104.83 & 8.50 & 0.05 & -104.48 & 9.44 \\ 36 \text { to } 47 & 0.00 & -111.80 & 22.45 & 0.00 & -108.16 & 16.79 \\ 48 \text { to } 59 & 0.00 & -112.65 & 24.13 & 0.00 & -111.86 & 24.19 \\ \geq 60 & 0.00 & -112.73 & 24.29 & 0.00 & -112.35 & 25.16\end{array}$

Node D: Edwards Plateau Eurycea

$\begin{array}{lllllll}0 \text { to } 11 & 0.64 & -100.58 & 0.00 & 0.61 & -99.37 & 0.00 \\ 12 \text { to } 23 & 0.35 & -102.10 & 3.04 & 0.36 & -103.36 & 7.97 \\ 24 \text { to } 35 & 0.01 & -108.61 & 16.06 & 0.03 & -106.49 & 14.2 \\ 36 \text { to } 47 & 0.00 & -112.55 & 23.95 & 0.00 & -111.76 & 24.78 \\ 48 \text { to } 59 & 0.00 & -112.769 & 24.37 & 0.00 & -112.64 & 26.53 \\ \geq 60 & 0.00 & -112.79 & 24.42 & 0.00 & -112.74 & 26.74\end{array}$

Node E: southern Edwards Plateau Eurycea

\begin{tabular}{lllllll}
0 to 11 & 0.32 & -101.58 & 0.90 & 0.34 & -102.28 & 0.77 \\
12 to 23 & 0.64 & -101.13 & 0.00 & 0.61 & -101.89 & 0.00 \\
24 to 35 & 0.04 & -107.75 & 13.23 & 0.05 & -106.87 & 9.95 \\
36 to 47 & 0.00 & -112.74 & 23.22 & 0.00 & -112.44 & 21.09 \\
48 to 59 & 0.00 & -112.80 & 23.34 & 0.00 & -112.78 & 21.77 \\
$\geq 60$ & 0.00 & -112.81 & 23.36 & 0.00 & -112.79 & 21.79 \\
\hline
\end{tabular}

Proportional probabilities (prob.) are from Bayesian reconstructions of six ordered maturation age categories ( 0 to 11,12 to 23,24 to 35,36 to 47,48 to 59 , and $\geq 60$ months) for males ( $M$; Figure 5 ) and females ( $F$; Figure 6). Model fitting comparisons were performed by fixing ('fossilizing') select nodes to the six alternative maturation age categories and comparing Log Bayes factors (LBf) to the lowest (best fitting) age category for the node based on the harmonic mean (hm).

mechanism of larval form paedomorphosis throughout this clade or if both mechanisms can occur [8]. It is also important to perform intra and interspecific experimental comparisons to test the role of plasticity in regulating these phenomena [8] (see also below).

Our original intention for this study was to test heterochonic patterns of spelerpine plethodontids, but in doing so we also show strong support for direct development as the ancestral life history for the family (Figure 2; Additional file 4). Previous tests of this pattern showed that the radiation of biphasic desmognathines is deeply nested among direct developing taxa [28]. However, some direct developing taxa (Hydromantes and then undiscovered Karsenia [39]) were not included in that study, and some important basal relationships were still unresolved. As a consequence, the ancestral life history for plethodontids was equivocal. Our support for direct development as the ancestral life history of plethodontids (as well as other deep nodes) suggests at least two or three reversals to biphasic life histories in plethodontids (biphasic desmognathines, spelerpines, and Hemidactylium). It also by default suggests that direct development may have a single origin in the family. This scenario for life history evolution in the Plethodontidae has major implications for understanding morphological evolution, particularly of larval forms [62-67]. To test such questions will require individual reconstructions of morphological traits in light of new phylogenetic hypotheses and perspectives on the evolution of developmental timing in this family.

\section{Considering plasticity of heterochronic changes?}

It is well established that environmental factors such as habitat desiccation, food availability, and temperature can influence the timing of metamorphosis and maturation in amphibians [68-71]. The potential effects of temperature on development also partly shaped previous heterochronic hypotheses [26]. This is because, in addition to the idea that ancestral plethodontids were large with long larval periods, it was also thought that the 'center of origin' of the family was the high elevations of the southern Appalachian Mountains [29-31,72]. This biogeographic pattern seemed consistent with the multiple independent accelerations in larval period. That is, larval development was accelerated as lineages dispersed from colder uplands to warmer lowlands. Temperature, food availability, and hydroperiod can influence the age of metamorphosis (and probably maturation) of plethodontids [9,73-77]. Nevertheless, these effects do not surpass differences among the most developmentally distinct lineages, such as the nearly 10 -fold difference in minimum age of metamorphosis between G. porphyriticus and E. quadridigiata. Stark differences in metamorphic age are also found in syntopy. For example, in the southern Appalachians, age at metamorphosis varies from 12 to 24 months for Eurycea wilderae and 36 to 60 months for Gyrinophilus porphyriticus, despite the fact that these species broadly overlap in distribution and develop in the same streams 


\section{Heterochronic Patterns of Paedomorphosis}
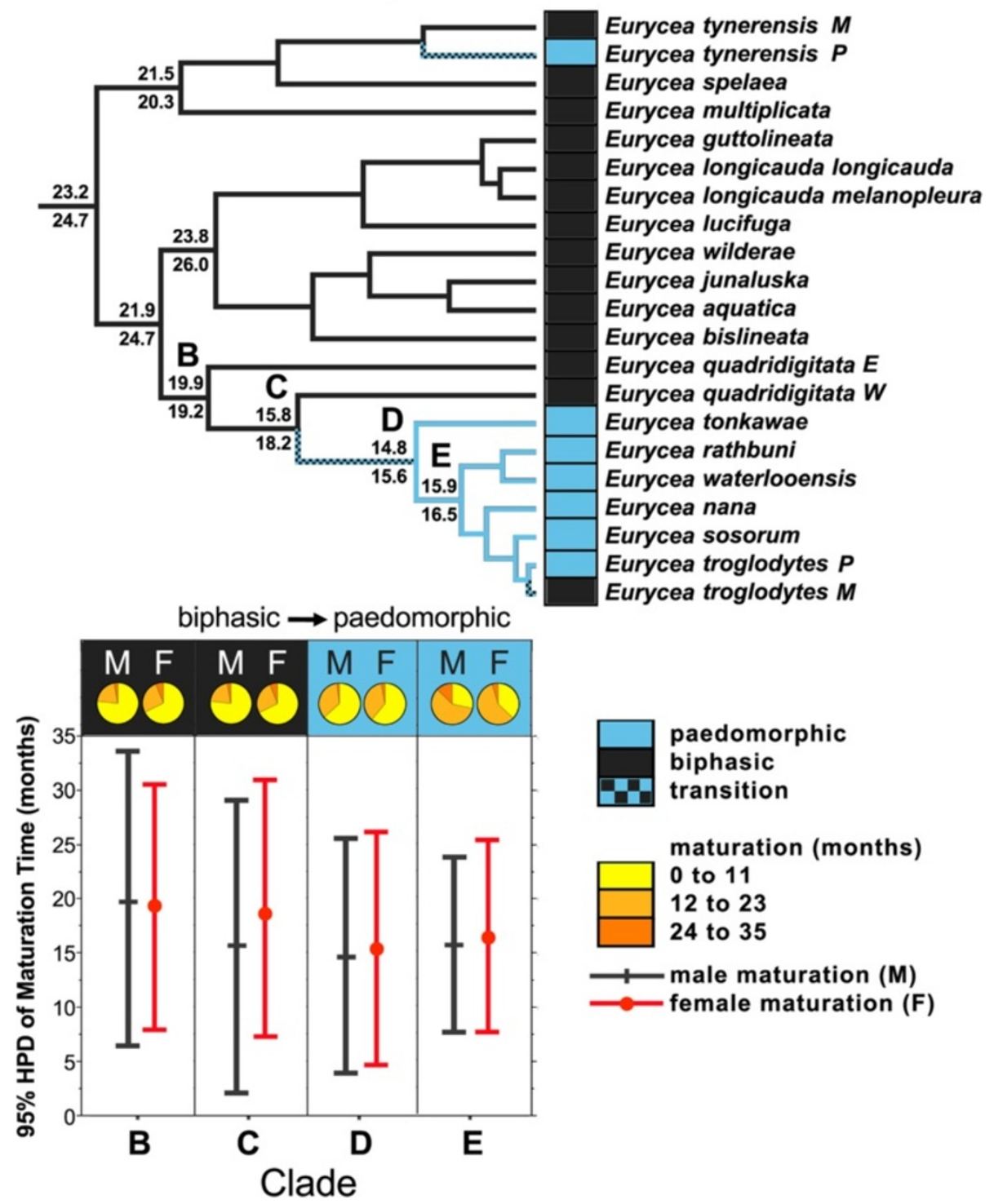

Figure 7 Evolution of paedomorphosis in a clade of Eurycea from the Edwards Plateau. The phylogeny shows the evolution of life history in spelerpines (biphasic vs. paedomorphic). The reconstruction is drawn from the ordered, three state analysis (Figure 2), and pruned to show only the taxa with maturation data (Figures 5 and 6). Four key nodes of interest are indicated (B to E), and a transitions from biphasic to paedomorphic likely occurred between nodes $C$ and D. Proportional probabilities subtending each node are Bayesian continuous estimates of maturation time for males (above; Figure 5) and females (below; Figure 6). Pie diagrams (also extrapolated from Figures 5 and 6), show categorical estimates for nodes B to E. Vertical lines on the graph indicate the average (symbol) and 95\% HPD intervals of continuous Bayesian estimates of male (black) and female (red) maturation age for the same nodes. These analyses do not provide evidence that larval form paedomorphosis in Edwards Plateau Eurycea (node C) evolved via progenesis, but rather indicate a case of neoteny. Major accelerations in metamorphosis and maturation likely evolved in an earlier ancestor (node B; Figures 3, 5, and 6), and early maturation age was simply maintained during the later shifts in somatic development (biphasic to paedomorphic; nodes C to D).

(Additional file 1). Likewise, the larval periods of $E$. quadridigitata (4 to 6.5 months) and Stereochilus marginatus (13 to 28 months) are distinctly different, yet these species occur and develop in the same habitats on the lowland Coastal Plain.
Here we analyzed minimum metamorphic ages across species, which strongly differ among plethodontids (Figures 3 and 4), and highlights a clear pattern; that the long larval periods of Gyrinophilus porphyriticus and Pseudotriton ruber are clearly derived decelerations. However, our model for 
spelerpines is certainly not fixed. Environmentally induced shifts in metamorphic age could certainly push some species to accelerate or decelerate metamorphic timing compared to ancestral spelerpines, or even close relatives. For example, larval Eurycea bislineata in some lakes as well as far northern populations, attain a very large body sizes, and the latter are known to take at least 3 years to metamorphose [78,79]. On the opposite end of the distribution, thyroid hormone treatments of 8-month-old, lab-raised Eurycea tynerensis larvae (from some populations) induce significant metamorphic changes which shows that metamorphic age can be accelerated physiologically [Bonett et al., unpublished]. Further metamorphic data are needed in two important areas: (1) direct comparisons of larvae raised under common conditions to test for more subtle evolutionary shifts in heterochronic patterns among closely related species or populations; and (2) experimental tests of the degree and limits of plasticity within and among species.

\section{Phylogenetic analyses of heterochronic patterns}

Accurate interpretations of ancestral states can have profound influences on understanding heterochronic patterns. This is because polarizing heterochronic analyses with distinctly different ancestral states can result in opposite reconstructions and interpretations of the pattern. Here we show examples of how: (1) reconstructing the ancestral timing of metamorphosis in spelerpines changes previous interpretations of patterns in the acceleration and deceleration of descending taxa (Figure 4), and 2) reconstructing maturation across a major life history transition from biphasic to paedomorphic in a clade of Eurycea supports a case of neoteny rather than progenesis (Figure 7).

Several methods for analyzing the evolution of heterochronic patterns have been developed over the past two decades. These methods have largely focused on 'sequence heterochronies', which seek to analyze the sequence of developmental changes of multiple traits among species via event-pairing (for example, [13-18,80]). Sequence heterochrony methods allow for analyses of individual elements and mixed data types, and alleviate the need for comparable data on age or size for developmental events [57]. Tracing the evolution of sequence heterochronies can also be a complex, and in some cases intractable, problem due to the high number of alternative ancestral sequences (character states) relative to the number of taxa analyzed. By comparison, 'growth heterochrony' (also known as deBeerian heterochrony) analyses, where size and shape are used as a proxy for age $[2,3,57,81]$, are relatively more straightforward to reconstruct. A continuous character (for example, absolute age, relative age, size, or shape) is used to code the timing of developmental events. Data for 'growth heterochronies' are not always available or comparable among taxa, and therefore phylogenetic-based methods for reconstructing developmental events based on age or size have received less attention than sequence heterochrony methods. In fact, few studies have incorporated error in ancestral estimates to test for significant heterochronic shifts in a phylogenetic context [for example, 17,19].

In our study, the data available and problem at hand allow us to directly analyze the age of metamorphosis and maturation among species. We used both categorical and continuous reconstructions in our analyses to directly test heterochronic changes with respect to age, and both coding schemes (and methods) have advantages and limitations. Categorical analyses facilitate explicit hypothesis tests to analyze the best fit of alternative ancestral states (that is, age categories [54]), which extends analyses beyond examining calculated probabilities of ancestral states. An obvious advantage of a continuous analysis is that variables do not have to be partitioned into categories, which can be somewhat arbitrary, and could mask shifts in ancestral states that fall within a category. We used 12month intervals to categorize numbers of months, and our categorical and continuous results largely agreed. Also, as we show here, variation in estimation of continuous ancestral states (for example Bayesian 95\% HPD intervals) can be used to assess shifts in developmental timing between nodes, or between nodes and tips. A limitation of reconstructing continuous characters is that lineages that have undergone major shifts (in the character of interest) may effect ancestral state estimation across the tree. This is because the common models used (for example, Brownian Motion and Ornstein-Uhlenbeck) are effectively averaging tip values across ancestral nodes and may not effectively account for major rate shifts within the tree $[82,83]$. Another challenge is that variation in ancestral state estimation, especially for deep nodes, can be broad, which may make it difficult to detect significant heterochronic shifts between ancestors and descendants [17]. Stable trait reconstruction methods are currently being developed that account for major rate shifts when estimating continuous ancestral states [84]. These methods should offer more accurate estimates of continuous traits with lower mean squared error than Brownian Motion [84], which should improve our ability to statistically detect subtle heterochronic shifts. Despite the need for further methodological improvements, we present additional methods and examples that demonstrate how ancestral state reconstruction can be employed to test patterns of heterochronic evolution.

\section{Conclusions}

Phylogenetic-based ancestral state reconstructions of metamorphic age (using both categorical and continuous coding) show that ancestral spelerpines had relatively shorter larval periods than previously suggested. This repolarization of the ancestral condition has major implications 
for understanding patterns of heterochrony in descending lineages. The long larval periods in a few taxa (for example, Gyrinophilus porphyriticus and Pseudotriton ruber) were likely derived decelerations (Figures 3 and 4), rather than a reflection of the ancestral condition. Only a few species/clades with very short larval periods (for example, Eurycea quadridigitata and E. longicauda) likely underwent accelerations in larval development. In contrast, most extant spelerpines have larval periods that are not discernably different than the ancestor for this clade (possibly the result of stasis). We also show that the evolution of paedomorphosis in an endemic radiation of Eurycea from the Edwards Plateau of central Texas was more likely the result of neoteny. This is because our reconstructions of ancestral maturation age show little change in the timing of maturation across the major delay in somatic morphogenesis leading to larval form paedomorphosis (Figure 7). There have been multiple independent instances of paedomorphosis in spelerpines (Figure 2), and more data on maturation times are needed to test if neoteny is a generalizable mechanism for the evolution of larval form paedomorphosis in this clade. Finally, in some systems, ancestral state reconstructions may be critical for testing ancestral conditions when polarizing patterns of heterochronic evolution.

\section{Additional files}

\section{Additional file 1: Table of taxa, developmental data, and Genbank accession numbers.}

Additional file 2: Table of primers and DNA sequencing methods. Additional file 3: Table of substitution models used in phylogenetic analyses.

Additional file 4: Categorical life history reconstructions including outgroup families.

Additional file 5: Additional categorical tests of timing of metamorphosis.

Additional file 6: Additional continuous reconstructions of metamorphosis and maturation.

\section{Competing interests}

The authors declare that they have no competing interests.

\section{Authors' contributions}

MAS, GAR, and RMB compiled the data. RMB performed analyses and drafted the manuscript. All authors contributed to the preparation of the manuscript. All authors read and approved the final manuscript.

\section{Acknowledgements}

We thank N. Bendik, D. Chamberlain, L. Dries, J. Fries, A. Gluesenkamp, and S. Trauth for information on developmental timing of several species of Eurycea, and C. Brown, A. Trujano, D. Wake, and two anonymous reviewers for comments on the study and manuscript. Specimens were handled in accordance with Institutional Animal Care and Use Committee (IACUC) protocols at the University of Tulsa (TU-0029). This study was in part funded by the National Science Foundation (DEB 1050322 to RMB; DEB1210859 to $\mathrm{RMB}$ and MAS).
Received: 27 May 2014 Accepted: 21 July 2014

Published: 18 August 2014

\section{References}

1. Gould SJ: Ontogeny and Phylogeny. Cambridge, MA: Harvard University Press; 1977.

2. Alberch P, Gould SJ, Oster GF, Wake DB: Size and shape in ontogeny and phylogeny. Paleobiology 1979, 5:296-317.

3. Raff RA, Wray GA: Heterochrony: developmental mechanisms and evolutionary results. J Evol Biol 1989, 2:409-434.

4. McKinney ML, McNamara KJ: Heterochrony, the Evolution of Ontogeny. New York, NY: Plenum Press; 1991.

5. Zelditch ML, Fink WM: Heterochrony and heterotopy: stability and innovation in the evolution of form. Paleobiology 1996, 22:241-254.

6. Wake DB, Larson A: Multidimensional analysis of an evolving lineage. Science 1987, 238:42-48.

7. Ryan TJ, Semlitsch RD: Intraspecific heterochrony and life history evolution: decoupling somatic and sexual development in a facultatively paedomorphic salamander. Proc Natl Acad Sci USA 1998, 95:5643-5648.

8. Denöel M, Joly P: Neoteny and progenesis as two heterochronic processes involved in paedomorphosis in Triturus alpestris (Amphibia: Caudata). Proc R Soc Lond Ser B 2000, 267:1481-1485.

9. Bruce RC: Theory of complex life cycles: application in plethodontid salamanders. Herpetol Monogr 2005, 19:180-207.

10. Buckley D, Alcobendas M, Garcia-Paris M, Wake MH: Heterochrony, cannibalism, and the evolution of viviparity in Salamandra salamandra. Evol Dev 2007, 9:105-115.

11. Fink WL: The conceptual relationship between ontogeny and phylogeny. Paleobiology 1982, 8:254-264

12. Klingenberg $C P$, Spence JR: Heterochrony and allometry: lessons from the water strider genus Limnoporus. Evolution 1993, 47:1834-1853.

13. Velhagen WAJ: Analyzing developmental sequences using sequences units. Syst Biol 1997, 46:204-210.

14. Nunn CL, Smith KK: Statistical analyses of developmental sequences: The craniofacial region in marsupial and placental mammals. Am Nat 1998, 152:82-101

15. Smith KK: Time's arrow: heterochrony and the evolution of development. Int J Dev Biol 2003, 47:613-621.

16. Jeffery JE, Bininda-Emonds ORP, Coates MI, Richardson MK: A new technique for identifying sequence heterochrony. Syst Biol 2005, 54:230-240.

17. Germain D, Laurin M: Evolution of ossification sequences in salamanders and urodele origins assessed through event-pairing and new methods. Evol Dev 2009, 11:170-190

18. Maxwell E, Harrison LB: Methods for the analysis of developmental sequence data. Evol Dev 2009, 11:109-119.

19. Geiger M, Forasiepi AM, Koyabu D, Sánchez-Villagra MR: Heterochrony and post-natal growth in mammals - an examination of growth plates in limbs. J Evol Biol 2013, 27:98-115.

20. Hanken J: Is heterochrony still an effective paradigm for contemporary studies of Evo-Devo? In Conceptual change in biology: scientific and philosophical perspectives on evolution and development. Edited by Love A. New York, NY: Springer-Verlag; 2014.

21. AmphibiaWeb: Information on Amphibian Biology and Conservation. Berkeley, CA: AmphibiaWeb; 2014 [http://amphibiaweb.org/]

22. Duellman WE, Trueb LT: Biology of Amphibians. Baltimore, MD: The Johns Hopkins University Press; 1986.

23. Hanken J: Life history and morphological evolution. J Evol Biol 1992, 5:549-557.

24. Wiens JJ, Bonett RM, Chippindale PT: Ontogeny discombobulates phylogeny: paedomorphosis and higher-level salamander relationships. Syst Biol 2005, 54:91-110.

25. Bonett RM, Steffen MA, Lambert SM, Wiens JJ, Chippindale PT: Evolution of paedomorphosis in plethodontid salamanders: ecological correlates and re-evolution of metamorphosis. Evolution 2014, 68:466-482.

26. Ryan TJ, Bruce RC: Life history evolution and adaptive radiation of hemidactyliine salamanders. In The Biology of Plethodontid Salamanders. Edited by Bruce RC, Jaeger RG, Houck LD. New York, NY: Kluwer Academic, Plenum Publishers; 2000:303-325.

27. Reilly SM, Wiley EO, Meinhardt D: An integrative approach to heterochrony: distinguishing intraspecific and interspecific phenomena. Biol J Linn Soc 1997, 60:119-143. 
28. Chippindale PT, Bonett RM, Baldwin AS, Wiens JJ: Phylogenetic evidence for a major reversal of life-history evolution in plethodontid salamanders. Evolution 2004, 58:2809-2822

29. Wilder IW, Dunn ER: The correlation of lunglessness in salamanders with a mountain brook habitat. Copeia 1920, 84:63-68.

30. Dunn ER: The Salamanders of the family Plethodontidae. Smith College: Northampton, MA; 1926.

31. Wake DB: Comparative osteology and evolution of the lungless salamanders, Family Plethodontidae. Mem So Cal Acad Sci 1966, 4:1-111.

32. Bruce RC: Variation in the life cycle of the salamander Gyrinophilus porphyriticus. Herpetologica 1972, 28:230-245.

33. Bruce RC: Life-history patterns of the salamander Gyrinophilus porphyriticus in the Cowee Mountains, North Carolina. Herpetologica 1978, 34:53-64.

34. Mueller RL, Macey JR, Jaekel M, Wake DB, Boore JL: Morphological homoplasy, life history evolution, and historical biogeography of plethodontid salamanders inferred from complete mitochondrial genomes. Proc Natl Acad Sci USA 2004, 101:13820-13825.

35. Kerney RR, Blackburn DC, Müller H, Hanken J: Do larval traits re-evolve? Evidence from the embryogenesis of a direct-developing salamander, Plethodon cinereus. Evolution 2012, 66:252-262.

36. Tilley SG: Life histories and comparative demography of two salamander populations. Copeia 1980, 1980:806-821.

37. Bruce RC: An explanation for differences in body size between two desmognathine salamanders. Copeia 1990, 1990:1-9.

38. Steffen MA, Irwin KJ, Blair AL, Bonett RM: Larval masquerade: a new species of paedomorphic salamander (Caudata: Plethodontidae: Eurycea) from the Ouachita Mountains of North America. Zootaxa 2014, 3786:423-442.

39. Min M-S, Yang SY, Bonett RM, Vieites DR, Brandon RA, Wake DB: Discovery of the first Asian plethodontid salamander. Nature 2005, 435:87-90.

40. Mueller RL: Evolutionary rates, divergence dates, and the performance of mitochondrial genes in Bayesian phylogenetic analysis. Syst Biol 2006, 55:289-300

41. Vieites DR, Min M-S, Wake DB: Rapid diversification and dispersal during periods of global warming by plethodontid salamanders. Proc Natl Acad Sci USA 2007, 104:19903-19907.

42. Kozak KH, Mendyk RW, Wiens JJ: Can parallel diversification occur in sympatry? Repeated patterns of body-size evolution in co-existing clades of North American salamanders. Evolution 2009, 63:1769-1784.

43. Pyron RA, Wiens JJ: A large-scale phylogeny of Amphibia including over 2,800 species, and a revised classification of extant frogs, salamanders, and caecilians. Mol Phylogent Evol 2011, 2011:543-583.

44. Wiens JJ, Engstrom TN, Chippindale PT: Rapid diversification, incomplete isolation, and the "speciation clock" in North American salamanders (genus Plethodon): testing the hybrid swarm hypothesis of rapid radiation. Evolution 2006, 60:2585-2603

45. Nylander JAA: MrModeltest v. 2.2. Uppsala: Evolutionary Biology Centre, Uppsala University; 2004

46. Drummond AJ, Rambaut A: BEAST: Bayesian evolutionary analysis by sampling trees. BMC Evol Biol 2007, 7:214.

47. Boardman GS, Schubert BW: First Mio-Pliocene salamander fauna from the southern Appalachians. Palaeontologia Electronica 2011, 14:16A

48. Holman JA: Fossil salamanders of North America. Bloomington, IN: Indiana University Press; 2006.

49. Wiens JJ: Global patterns of species richness and diversification in amphibians. Am Nat 2007, 170:S86-S106.

50. Roelants K, Gower DJ, Wilkinson M, Loader SP, Biju SD, Guillaume K, Moriau $\mathrm{L}$, Bossuyt F: Global patterns of diversification in the history of modern amphibians. Proc Natl Acad Sci USA 2007, 104:887-892

51. Zhang $P$, Wake DB: Higher-level salamander relationships and divergence dates inferred from complete mitochondrial genomes. Mol Phylogenet Evol 2009, 53:492-508.

52. Rambaut A, Drummond AJ: Tracer v. 1.5. ; 2007 [http://beast.bio.ed.ac.uk] Tracer]

53. Pagel M, Meade A: BayesTraits v. 2.0. Reading: University of Reading; 2013 [http://www.evolution.rdg.ac.uk]

54. Pagel M, Meade A, Barker D: Bayesian estimation of ancestral character states on phylogenies. Syst Biol 2004, 53:673-684.

55. Burnham KB, Anderson D: Model Selection and Multi-Model Inference: A practical Information Theoretic Approach. 2nd edition. New York, NY: Springer-Verlag; 2002.
56. Revell LJ: Phytools: Phylogenetic Tools for Comparative Biology. Boston, MA: University of Massachusetts; 2012. http://faculty.umb.edu/liam.revell/ phytools/

57. Smith KK: Heterochrony revisited: the evolution of developmental sequences. Biol J Linn Soc 2001, 73:169-186.

58. Bruce RC: Evolution of paedomorphosis in salamanders of the genus Gyrinophilus. Evolution 1979, 33:998-1000.

59. Bruce RC: Population structure, life history, and evolution of paedogenesis in the salamander Eurycea neotenes. Copeia 1976, 1976:242-249.

60. Sweet SS: Natural metamorphosis in Eurycea neotenes and the generic allocation of Texas Eurycea (Amphibia; Plethodontidae). Herpetologica 1977, 33:364-375

61. Lamb T, Beamer DA: Digits lost or gained? Evidence for pedal evolution in the dwarf salamander complex (Eurycea, Plethodontidae). PLOS ONE 2012, 7:e37544.

62. Collazo A, Marks SB: Development of Gyrinophilus porphyriticus: Identification of the ancestral developmental pattern in the salamander family Plethodontidae. J Exp Zoo/ 1994, 268:239-258.

63. Wake DB, Hanken J: Direct development in the lungless salamanders: what are the consequences for developmental biology, evolution, and phylogenesis? Int J Dev Biol 1996, 40:859-869.

64. Marks SB, Collazo A: Direct development in Desmognathus aeneus (Caudata: Plethodontidae): a staging table. Copeia 1998, 1998:637-648.

65. Marks SB: Skull development in two plethodontid salamanders (genus Desmognathus) with different life histories. In The biology of plethodontid salamanders. Edited by Bruce RC, Jaeger RG, Houck LD. New York, NY: Kluwer Academic, Plenum Publishers; 2000:261-276.

66. Deban SM, Marks SB: Metamorphosis and evolution of feeding behavior in salamanders of the family Plethodontidae. Zool J Linnean Soc 2002 134:375-400.

67. Rose CS: The developmental morphology of salamander skulls. In Amphibian biology, Vol. 5. Osteology. Edited by Heatwole H, Davies M. Baulkham Hills BC: Surrey Beatty and Sons Pty. Ltd; 2003:1686-1783.

68. Wilbur HM, Collins JP: Ecological aspects of amphibian metamorphosis. Science 1973, 182:1305-1314.

69. Semlitsch RD, Wilbur HM: Effects of pond drying time on metamorphosis and survival in the salamander Ambystoma talpoideum. Copeia 1988, 1988:978-983.

70. Denver RJ, Mirhadi N, Phillips M: Adaptive plasticity in amphibian metamorphosis: response of Scaphiopus hammondii tadpoles to habitat desiccation. Ecology 1998, 79:1859-1872.

71. Newman RA: Ecological constraints on amphibian metamorphosis: interactions of temperature and larval density with responses to changing food level. Oecologia 1998, 115:9-16.

72. Beachy CK, Bruce RC: Lunglessness in plethodontid salamanders is consistent with the hypothesis of a mountain stream origin: a response to Ruben and Boucot. Am Nat 1992, 139:839-847.

73. Voss SR: The relationship between stream order and length of larval period in the salamander Eurycea wilderae. Copeia 1993, 1993:736-742.

74. Beachy CK: Effects of larval growth history on metamorphosis in a stream-dwelling salamander (Desmognathus ochrophaeus). J Herpetol 1995, 29:375-382

75. Camp CD, Marshall JL, Austin RM Jr: The evolution of adult body size in black-bellied salamanders (Desmognathus quadramaculatus complex). Can J Zool 2000, 78:1712-1722.

76. Freeman SL, Bruce RC: Larval period and metamorphosis of the three-lined salamander, Eurycea guttolineata (Amphibia: Plethodontidae), in the Chattooga River watershed. Am Midl Nat 2001, 145:194-200.

77. Hickerson C-AM, Barker EL, Beachy CK: Determinants of metamorphic timing in the blackbellied salamander, Desmognathus quadramaculatus. Southeast Nat 2005, 4:33-50.

78. Trapido H, Clausen RT: The larvae of Eurycea bislineata major. Copeia 1940, 1940:244-246.

79. Bahret R: Ecology of lake dwelling Eurycea bislineata in the Shawangunk Mountains, New York. J Herpetol 1996, 30:399-401.

80. Andrews RM, Brandley MC, Greene WW: Developmental sequences of squamate reptiles are taxon specific. Evol Dev 2013, 5:326-343.

81. Wake MH, Hanken J: Development of the skull of Dermophis mexicanus (Amphibia: Gymnophiona) with comments on skull kinesis and amphibian relationships. J Morph 1982, 173:203-223. 
82. Slater G, Harmon L, Alfaro M: Integrating fossils with molecular phylogenies improves inference of trait evolution. Evolution 2012, 66:3931-3944.

83. Slater GL: Phylogenetic evidence for a shift in the mode of mammalian body size evolution at the Cretaceous-Palaeogene boundary. Methods Ecol Evol 2013, 4:734-744.

84. Elliot MG, Mooers $A \varnothing$ : Inferring ancestral states without assuming neutrality or gradualism using a stable model of continuous character evolution. 2013, arXiv:1302.5104.

doi:10.1186/2041-9139-5-27

Cite this article as: Bonett et al:: Heterochrony repolarized: a

phylogenetic analysis of developmental timing in plethodontid salamanders. EvoDevo 2014 5:27.

\section{Submit your next manuscript to BioMed Central and take full advantage of:}

- Convenient online submission

- Thorough peer review

- No space constraints or color figure charges

- Immediate publication on acceptance

- Inclusion in PubMed, CAS, Scopus and Google Scholar

- Research which is freely available for redistribution 